\title{
Commentary The genetics of acute lung injury: looking back and pointing the way forward Ednan K Bajwa
}

Pulmonary and Critical Care Unit, Massachusetts General Hospital, Harvard Medical School, Bulfinch 148, 55 Fruit Street, Boston, MA 02114, USA

Corresponding author: Ednan K Bajwa, ebajwa@partners.org

Published: 12 January 2009

This article is online at http://ccforum.com/content/13/1/108

(c) 2009 BioMed Central Ltd

See related research by Flores et al., http://ccforum.com/content/12/6/R130
Critical Care 2009, 13:108 (doi:10.1186/cc7132)

\begin{abstract}
Individual genetic factors have long been suspected of playing a major role in susceptibility to acute lung injury and acute respiratory distress syndrome. Flores and colleagues evaluate the quality of published studies testing the relationships between variation in candidate genes and susceptibility to lung injury syndromes or worsened outcome in patients with these conditions. Their results demonstrate that while important advances have been made in this area, attention should be paid to improving the methodology of future studies in order to minimize the chances of publishing falsepositive results.
\end{abstract}

In the previous issue of Critical Care, Dr Flores and colleagues travel down a familiar path [1]. Throughout the history of medicine, physicians have wondered how to account for the fact that individuals may respond so differently to similar illnesses. Indeed, some of these relationships are obvious - patients who do not receive antibiotics in a timely fashion may progress rapidly from infection to septic shock [2]; an older patient may be more likely to die than a younger, healthier patient with the same illness [3]. Many a physician, however, has been left to wonder why, given two relatively similar patients with community-acquired pneumonia, one patient may recover quickly with a standard course of antibiotics while another patient develops catastrophic acute lung injury with its attendant profound hypoxemia, multiorgan failure, and high mortality rate.

The difference, of course, has long been thought to be in the genes. That is, the disease phenotype of the patient results from the sum of the complex interactions between the environment and the patient's genotype [4]. We are now at the beginning of the second decade of the effort to better define the role played by genetic variation in the pathogenesis of acute lung injury and its most severe form, acute respiratory distress syndrome [1].

The reasons for conducting such investigations are compelling. By establishing the importance of genetic variation in determining the final disease phenotype, it may be possible to work backwards and identify the proteins that play a more important role in disease pathogenesis. This might improve our understanding of acute lung injury/acute respiratory distress syndrome disease processes as well as generate targets for therapeutic intervention. From the standpoint of the clinician, knowing how the patient's genotype will affect the course of their illness might enable more accurate prognostic assessment, or may even allow targeted therapeutic interventions based on how the patient's genotype predicts they will respond [5].

As Flores and colleagues note, however, the road to these anticipated outcomes has been bumpy. In their comprehensive exploration of the state of the literature, the authors evaluated 29 original research studies that reported positive associations between genetic variants and an altered level of risk of developing acute lung injury/acute respiratory distress syndrome, or positive associations with clinical outcomes [1]. They then sought to evaluate the quality of these studies according to the recommendations of an expert panel [6]. This panel aimed to address a major issue that has plagued genetic association studies: replication validity. Despite a growing number of reported positive genotype-phenotype associations across a variety of different diseases, a substantial proportion of these associations lose significance when tested in different patient populations, casting doubt on whether the association truly exists or merely represents a chance finding [7]. As part of this group's recommendations, they developed a list of consensus criteria for evaluating the quality of studies that report novel associations. This is relevant because the use of less robust methodology in initial studies may hamper efforts to replicate their results [8]. The panel's criteria address a number of study design elements, including power and sample size, precise definition of phenotypes, careful steps to reduce confounding, appropriate selection of statistical methods, and close attention to quality control. 
Using elements of these criteria as a guide, Flores and colleagues discovered a few notable trends in the published literature [1]. Most of the published studies reported positive associations, in a total of 16 genes. Of these positive associations, less than one-half were replicated in additional populations. It is impossible to know how many of the unreplicated results were not published because the associations were found to be negative, or how many additional genes showed negative associations that were not published. Many studies had disappointing sample sizes, with a median of around 100 to 200 patients depending on the study design. In addition, most studies did not report power calculations. Flores and colleagues also point out heterogeneity in the selection of a control group for case-control studies, with a plurality of the studies selecting healthy subjects or population-based control individuals.

These findings present a mixed picture - owing to the number of studies with small sample sizes, a lack of replication studies, absence of power calculations, and an unknown number of unpublished negative studies, it is possible that many of these associations were found merely due to chance. The choice of control groups in some studies is also somewhat troubling. If the goal is to study the impact of genotype on the risk of developing lung injury, it seems counterintuitive to select healthy patients or population-based control individuals as the comparison group since these patients may in fact be genetically susceptible and only require an appropriate external stimulus to develop the disease.

Despite these potential flaws in published studies, the authors also point to successes in the literature that can be built upon - they suggest a need for studies with improved initial quality (large sample sizes with adequate power, selection of appropriate control groups, use of functional surrogates to support associations with disease phenotype) as well as the need for replication of initial findings. Future studies should also employ newer technologies and strategies such as large-scale multigene association studies, gene expression studies [9], and biomarker/proteomic technologies [10] both for identification of novel candidate genes and to provide additional data that support the relevance of reported associations. A concerted effort by researchers and journal editors should also be made to ensure that negative initial and replication studies are published to reduce the impact of publication bias. Finally, further studies in different racial populations are needed.

A decade into the course of this work, we have taken only small steps towards the goal. As Flores and colleagues show, however, the foundation has been laid for ultimately answering the crucial question posed by physicians - can we take what we have learned about genotypes and apply it to the care of the patient in front of us?

\section{Competing interests}

The author declares that they have no competing interests.

\section{References}

1. Flores C, Del Mar Pino-Yanes M, Villar J: A quality assessment of genetic association studies supporting susceptibility and outcome in acute lung injury. Crit Care 2008, 12:R130.

2. Garnacho-Montero J, Aldabo-Pallas T, Garnacho-Montero C, Cayuela A, Jimenez R, Barroso S, Ortiz-Leyba C: Timing of adequate antibiotic therapy is a greater determinant of outcome than are TNF and IL-10 polymorphisms in patients with sepsis. Crit Care 2006, 10:R111.

3. Gong MN, Thompson BT, Williams P, Pothier L, Boyce PD, Christiani DC: Clinical predictors of and mortality in acute respiratory distress syndrome: potential role of red cell transfusion. Crit Care Med 2005, 33:1191-1198.

4. Grumet FC, Coukell A, Bodmer JG, Bodmer WF, McDevitt HO: Histocompatibility (HL-A) antigens associated with systemic lupus erythematosus. A possible genetic predisposition to disease. N Engl J Med 1971, 285:193-196.

5. Martinez-Forero I, Pelaez A, Villoslada P: Pharmacogenomics of multiple sclerosis: in search for a personalized therapy. Expert Opin Pharmacother 2008, 9:3053-3067.

6. Chanock SJ, Manolio T, Boehnke M, Boerwinkle E, Hunter DJ, Thomas G, Hirschhorn JN, Abecasis G, Altshuler D, Bailey-Wilson JE, Brooks LD, Cardon LR, Daly M, Donnelly P, Fraumeni JF Jr, Freimer NB, Gerhard DS, Gunter C, Guttmacher AE, Guyer MS, Harris EL, Hoh J, Hoover R, Kong CA, Merikangas KR, Morton CC, Palmer LJ, Phimister EG, Rice JP, Roberts J, Rotimi C, Tucker MA, Vogan KJ, Wacholder S, Wijsman EM, Winn DM, Collins FS: Replicating genotype-phenotype associations. Nature 2007, 44:655-660.

7. Ioannidis JP, Ntzani EE, Trikalinos TA, Contopoulos-loannidis DG: Replication validity of genetic association studies. Nat Genet 2001, 29:306-309.

8. Hirschhorn JN, Lohmueller K, Byrne E, Hirschhorn K: A comprehensive review of genetic association studies. Genet Med 2002, 4:45-61.

9. Wurfel MM: Microarray-based analysis of ventilator-induced lung injury. Proc Am Thorac Soc 2007, 4:77-84.

10. Chang DW, Hayashi S, Gharib SA, Vaisar T, King ST, Tsuchiya M, Ruzinski JT, Park DR, Matute-Bello G, Wurfel MM, Bumgarner R, Heinecke JW, Martin TR: Proteomic and computational analysis of bronchoalveolar proteins during the course of the acute respiratory distress syndrome. Am J Respir Crit Care Med 2008, 178:701-709. 